\begin{tabular}{|c|c|}
\hline Title & Effects of surface treatment on Fermi level pinning at metal $G$ aN interfaces formed on homoepitaxial GaN lay ers \\
\hline Author(s) & Isobe, Kazuki; A kazawa, Masamichi \\
\hline Citation & $\begin{array}{l}\text { Japanese Journal of A pplied Physics (JJA P), 59(4), } 046506 \\
\text { https://doi.org/10.35848/1347-4065/ab8024 }\end{array}$ \\
\hline Issue Date & $2020-04-01$ \\
\hline Doc URL & http:/hdl .handle.net/2115/80770 \\
\hline Rights & ( $) 2020$ The Japan Society of A pplied Physics \\
\hline Type & article (author version) \\
\hline File Information & I sobe_JJAP59_046506_HUSCAP.pdf \\
\hline
\end{tabular}

Instructions for use 


\title{
Effects of Surface Treatment on Fermi Level Pinning at Metal/GaN Interfaces Formed on Homoepitaxial GaN Layers
}

\author{
Kazuki Isobe and Masamichi Akazawa* \\ Research Center for Integrated Quantum Electronics, Hokkaido University, Sapporo \\ 060-0813, Japan \\ *E-mail: akazawa@rciqe.hokudai.ac.jp
}

The effect of chemical surface treatment on the uncontrolled surface oxide at a GaN surface and on Fermi level pinning at subsequently formed metal/GaN interfaces was investigated for a GaN epitaxial layer grown on a GaN substrate. The impact of several chemical treatments, including photolithography, on the surface oxide and the resultant surface band bending at the GaN surface was examined by X-ray photoelectron spectroscopy. Surface band bending was reduced by the reduction in the amount of uncontrolled surface oxide. The metal/GaN interfaces formed subsequent to these chemical treatments were investigated by electrical measurement for Schottky barrier diodes. We found that the reduction in the amount of uncontrolled surface oxide leads to an increase in the slope factor in the metal-work-function dependence of the Schottky barrier height. The mechanism of Fermi level pinning at the metal/GaN interface is discussed. 


\section{Introduction}

$\mathrm{GaN}$ is a promising material for power devices ${ }^{1,2)}$ because of its wide band gap $(3.4 \mathrm{eV})$, high breakdown field $(3.3 \mathrm{MV} / \mathrm{cm})$, high electron mobility $\left(1,200 \mathrm{~cm}^{2} / \mathrm{V} \cdot \mathrm{s}\right)$, and high electron saturation velocity $\left(2.6 \times 10^{7} \mathrm{~cm} / \mathrm{s}\right)$. High-quality $\mathrm{GaN}$ epitaxial layers can be obtained since GaN free-standing substrates have recently become available, enhancing the expectation that GaN can be useful for high-efficiency power devices. To fully derive the excellent characteristics of GaN-on-GaN epitaxial layers, control of the surface and interface is a critical issue. Surface treatment can affect the characteristics of subsequently formed interfaces with different materials. Since a metal/GaN interface is one of the most important building blocks in electronic devices, it is necessary for device design to obtain knowledge about the effect of surface treatment on the electrical properties of this interface.

The Schottky barrier height, $\phi_{\mathrm{B}}$, is a key parameter in characterizing the metal/GaN interface. According to the Schottky-Mott model ${ }^{3)}, \phi_{\mathrm{B}}$ at an ideal metal/semiconductor interface is given by

$$
\phi_{\mathrm{B}}=\phi_{\mathrm{M}}-\chi
$$

where $\phi_{\mathrm{M}}$ is the work function of the metal and $\chi$ is the electron affinity of the semiconductor. However, in general, this relation does not apply and the slope factor given by the following equation is used to describe the dependence of $\phi_{\mathrm{B}}$ on $\phi_{\mathrm{M}}$.

$$
S=\frac{d \phi_{\mathrm{B}}}{d \phi_{\mathrm{M}}}
$$

The $\phi_{\mathrm{B}}-\phi_{\mathrm{M}}$ relation with $S=1$ is called the Schottky limit, whereas that with $S=0$ is the Bardeen limit where the Fermi level is completely pinned, as shown in Fig. 1. Usually, $S$ takes a value smaller than 1 . This phenomenon is called Fermi level pinning, in a broad sense, at the metal/semiconductor interface. Stronger pinning results in a smaller $S$. Thus far, several models concerning the origin of this phenomenon have been proposed ${ }^{4-8}$. However, the mechanism of this phenomenon is still under debate.

For GaN-on-sapphire and GaN-on-SiC epitaxial layers, several reports have been published on the $\phi_{\mathrm{B}}-\phi_{\mathrm{M}}$ relation and the slope factor at the metal/GaN interface ${ }^{9-14)}$. However, these epitaxial layers have usually suffered from the leakage current under 
reverse bias voltage ${ }^{15)}$. The thin surface barrier (TSB) model has been proposed to explain the mechanism of this leakage current ${ }^{16,17)}$. According to the TSB model, leakage current flows through the thin surface tunneling barrier generated by the unintentional introduction of high-density donor-type defects. Recently, it has been reported that a GaN-on-GaN epitaxial layer is free from this kind of leakage current ${ }^{18,19)}$. Therefore, it is worth studying Fermi level pinning at metal/GaN interfaces for this layer. Indeed, the $\phi_{\mathrm{B}}-\phi_{\mathrm{M}}$ relation has been newly reported ${ }^{20-22)}$.

Surface treatment of air-exposed GaN prior to metal deposition is crucial for obtaining desirable electrical properties of a metal/GaN interface for the following reason. After crystal growth, the GaN surface is exposed to air, resulting in the inevitable formation of an uncontrolled surface oxide layer. Although several reports on surface treatment have been published, it is difficult to remove this oxide layer completely using ordinary solutions ${ }^{22-27)}$. Recently, it has been reported that the surface oxide layer formed by long-term air exposure after specific chemical treatments has a crystalline nature ${ }^{28-30)}$. However, even this kind of oxide layer can consist of multiple crystalline forms with disordered phases and Ga-related defects ${ }^{28,30)}$. Therefore, an uncontrolled surface oxide layer may be a source of disorder at the $\mathrm{GaN}$ surface and metal/GaN interfaces, although a "well-controlled" native oxide layer has been reported to reduce the interface state density at the insulator/GaN interface ${ }^{31-33)}$. According to the disorder-induced gap state (DIGS) model $^{8)}$, the origin of Fermi level pinning at the surface and interfaces is the gap states induced by disorder in chemical bonding. There is a possibility that the uncontrolled surface oxide layer causes Fermi level pinning at metal/GaN interfaces. It is important to know how this uncontrolled surface oxide layer affects the metal/GaN interface.

In this work, we investigated the impact of surface treatment on the uncontrolled surface oxide and on the electrical properties of the metal/GaN interfaces of a GaN-on-GaN epitaxial layer. The amount of surface oxide was varied by several chemical treatments, and the resultant Fermi level pinning at the subsequently formed metal/GaN interfaces was examined. We found that there is a correlation between the chemically treated $\mathrm{GaN}$ surface and the subsequently formed metal/GaN interfaces.

\section{Preparation of samples}

An n-type GaN epitaxial layer $\left(2 \mu \mathrm{m}\right.$, Si-doped: $\left.5.0 \times 10^{16} \mathrm{~cm}^{-3}\right)$ was grown by metalorganic vapor phase epitaxy on a free-standing c-plane n-type GaN substrate (grown by hydride vapor phase epitaxy, dislocation density $<5.0 \times 10^{6} \mathrm{~cm}^{-2}$, Si-doped: $2.0 \times 10^{18}$ 
$\mathrm{cm}^{-3}$ ). The effects of chemical surface treatments on the GaN surface were investigated by X-ray photoelectron spectroscopy (XPS) with a monochromated Al-Ka X-ray source $(1486.6 \mathrm{eV})$. For electrical characterization of the metal/GaN interfaces, Schottky barrier diodes (SBDs) were fabricated by forming metal electrodes onto the chemically treated GaN surface. To set the same surface treatment conditions for the sample subjected to surface investigation and the SBDs, we prepared the sample as follows. After organic cleaning, photolithography process including spin coating of photoresist, prebaking at $90^{\circ} \mathrm{C}$, exposure to deep-ultraviolet light, and a development process, was performed. Here, the developer solution mainly contained tetramethyl ammonium hydroxide (TMAH). Thereafter, a hydrochloric acid $(\mathrm{HCl})$ treatment $\left(\mathrm{HCl}: \mathrm{H}_{2} \mathrm{O}=1: 3,1 \mathrm{~min}\right)$ and a buffered hydrofluoric acid (BHF) treatment ( $\left.\mathrm{HF}: \mathrm{NH}_{4} \mathrm{~F}=1: 5,1 \mathrm{~min}\right)$ were performed, followed by rinsing with deionized water. It has been reported that $\mathrm{HCl}$ and $\mathrm{BHF}$ treatments are effective for reducing the amount of oxide on the GaN surface ${ }^{24-27)}$. We examined the effects of six treatments, as shown in Table I, by XPS. For the SBDs, Ag, Cu, Au, Ni, and Pt electrodes were subsequently deposited by electron-beam evaporation. The completed SBD sample structure is shown in Fig. 2.

\section{Results and discussion}

\subsection{Impact of surface treatment on $\mathrm{GaN}$ surface}

The O 1s spectra obtained by XPS for GaN layers with various surface treatments are summarized in Fig. 3 where the intensity is normalized to Ga 3d spectral area for each treatment so that the spectral area ratio of $\mathrm{O} 1 \mathrm{~s}$ to $\mathrm{Ga} 3 \mathrm{~d}$ is proportional to area of $\mathrm{O} 1 \mathrm{~s}$ spectrum in this figure. These spectra can be commonly decomposed into two components, i.e., the $\mathrm{O}-\mathrm{Ga}$ bonding component $(531 \mathrm{eV})$ and the $\mathrm{O}-\mathrm{H}$ bonding component $(533 \mathrm{eV})^{23)}$. The former is assigned to the surface oxide, the latter to adsorbed water molecules. It can be clearly seen that the intensity of the O-Ga component was reduced by BHF (treatment B) and $\mathrm{HCl}$ (treatment $\mathrm{C}$ ) treatments. Therefore, these treatments are efficient in reducing the amount of the surface native oxide. From the peak intensity, the thickness of the surface oxide layer after the $\mathrm{BHF}$ and $\mathrm{HCl}$ treatments is $0.1 \mathrm{~nm}$ or less, which indicates that the native oxide did not form a layer but was most likely scattered on the surface. Actually, the shapes of $\mathrm{Ga} 3 \mathrm{~d}$ spectra measured after these treatments coincide with that of the untreated surface, as shown in Fig. 4, which indicates that the oxide component was negligible in this spectrum because the surface oxide layer was very thin. Although the intensity of the O-Ga component was also reduced by dipping in the developer solution for 
$25 \mathrm{~s}$ (treatment A), it increased after a longer developing time of 1 min (treatment D). This result might indicate that the native oxide was regrown by developing for a longer time. Since the developer solution contained TMAH as the main component, it is highly likely that the surface of $\mathrm{GaN}$ was slightly etched during the developing process. This is natural if we consider that TMAH is used as an etchant for $\mathrm{GaN}^{34)}$.

With band bending, the surface Fermi level position is different from that in the bulk. When the doping concentration is sufficiently low to make the depletion layer width much larger than the photoelectron escape depth, the surface Fermi level position can be derived by XPS. The measured valence band maximum (VBM) spectrum after each surface treatment is shown in Fig. 5. It can be seen that the surface treatment shifted the VBM spectra, which indicated that the surface Fermi level position was shifted by the surface treatment. Referring to Fig. 3, it seems that the surface Fermi level positions are related to the intensity of the $\mathrm{O}-\mathrm{Ga}$ component of the $\mathrm{O}$ 1s spectrum. The Fermi level positions derived by straight-line extrapolation of the VBM spectra for several treatments are plotted versus the ratio of the $\mathrm{O} 1 \mathrm{~s} \mathrm{O}-\mathrm{Ga}$ bonding peak area $\left(\mathrm{IO}_{\mathrm{s}(\mathrm{O}-\mathrm{Ga})}\right)$ to the $\mathrm{Ga} 3 \mathrm{~d}$ spectral area ( $\left.I_{\mathrm{Ga3d}}\right)$ in Fig. 6, where solid circles indicate the experimental data and the solid line indicates the fitted line described later. It is clear that the surface Fermi level, $E_{\mathrm{FS}}$, approaches the conduction band minimum, $E_{\mathrm{C}}$, as the $I_{\mathrm{O} 1 \mathrm{~s}(\mathrm{O}-\mathrm{Ga})} / I_{\mathrm{Ga}}$ d ratio decreases. In other words, band bending was reduced as the amount of surface oxide decreased. Given the values of $E_{\mathrm{FS}}-E_{\mathrm{C}}$, the density of the space charge in the depletion layer $Q_{\mathrm{D}}$ can be derived by

$$
\frac{Q_{\mathrm{D}}}{q}=\sqrt{\frac{2 \varepsilon_{S} N_{D} \psi_{S}}{q}}=\sqrt{\frac{2 \varepsilon_{S} N_{D}\left(E_{F S}-E_{F B}\right)}{q}}
$$

where $q$ is the elemental charge, $\varepsilon \mathrm{s}$ is the dielectric constant of $\mathrm{GaN}, N_{\mathrm{D}}$ is the doping concentration, $\psi \mathrm{s}$ is the surface potential, and $E_{\mathrm{FB}}$ is the bulk Fermi level. This $Q_{\mathrm{D}}$ should have been compensated by the surface charge $Q_{\mathrm{s}}$ including the extrinsic fixed charge, the surface polarization charge, and the surface state charge. Therefore, we obtain

$$
\frac{Q_{\mathrm{S}}}{q}=-\frac{Q_{\mathrm{D}}}{q}
$$

- $Q_{\mathrm{S}} / q$ calculated from each $E_{\mathrm{FS}}$ position versus the $I_{\mathrm{O} 1 \mathrm{~s}(\mathrm{O}-\mathrm{Ga})} / I_{\mathrm{Ga}}$ d ratio is plotted by the solid circles in Fig. 7. In the semilog plot, a linear trend can be seen. The solid line in Fig. 
7 indicates the straight line fitted to the solid circles. Using this relation obtained by fitting, the relation between $E_{\mathrm{FS}}-E_{\mathrm{FB}}$ and $I_{\mathrm{O} 1 \mathrm{~s}(\mathrm{O}-\mathrm{Ga})} / I_{\mathrm{Ga} d}$ was calculated with Eqs. (3) and (4) as plotted by the solid line in Fig. 6. An excellent fit can also be seen in Fig. 6. These results indicated that the reduction in the amount of the oxide component leads to the reduction of the surface charge and band bending.

Band bending can be a result of the surface states and/or the fixed charge. With the obtained results, we cannot distinguish which is the cause of band bending. Still, the obtained results might indicate that the surface oxide caused the surface disorder leading to the generation of the surface states. There is a possibility that the reduction in amount of the surface oxide with the reduction of disorder leads to a reduction in Fermi level pinning at the metal/GaN interface after the deposition of the metal layers onto the chemically treated GaN surface.

\subsection{Fermi level pinning at metal/GaN interface}

Metal layers were deposited after performing the surface treatments on the GaN surface to fabricate SBDs. The current-voltage $(I-V)$ characteristics measured at RT are shown in Fig. 8 for SBDs with treatments A, B, C, and E. The formation of Ag electrodes on the GaN surface with treatment B was difficult, presumably because of the absorbed fluorine atoms on the surface. Therefore, the Ag-electrode SBD with treatment B is not included. SBDs with treatment $\mathrm{D}$ were not fabricated because no improvement of surface Fermi level pinning was seen compared with the case of treatment A. It can be seen that the variation of the magnitude of reverse-bias current is the smallest for treatment A and the largest for treatment $\mathrm{C}$.

To evaluate the Schottky barrier height, it is necessary to consider that the current transport mechanism depends on the carrier concentration and temperature ${ }^{35)}$. In other words, there is an applicable bias range for the current transport model that depends on the carrier concentration and temperature. On the basis of the calculation for the present samples at RT taken from Ref. 35, the thermionic emission (TE) model can be applied to the forward $I-V$ characteristics, while the thermionic-field emission (TFE) model is suitable for the reverse bias range.

In the TE model, $I-V$ characteristics are given by ${ }^{3)}$

$$
J=A^{*} T^{2} \exp \left(-\frac{q \phi_{B i}}{k T}\right)\left[\exp \left(\frac{q V}{n k T}\right)-1\right]
$$




$$
\begin{aligned}
& A^{*}=\frac{4 \pi q m^{*} k^{2}}{h^{3}}, \\
& \phi_{B}=\phi_{B i}+\Delta \phi, \\
& \Delta \phi=\left(\frac{q^{3} N_{D}\left|\psi_{S}\right|}{8 \pi^{2} \varepsilon_{S}^{3}}\right)^{\frac{1}{4}},
\end{aligned}
$$

where $A^{*}$ is the Richardson constant, $T$ is temperature, $q$ is the elementary charge, $\phi_{\mathrm{Bi}}$ is the Schottky barrier height lowered by the image force, $k$ is the Boltzmann constant, $n$ is the ideality factor, $m^{*}$ is the effective mass of an electron, $h$ is the Planck constant, and $\Delta \phi$ is the lowering of the barrier height due to the image force. In Fig. 9, examples of fitting results are shown for samples with (a) treatment A and (b) treatment C. For both treatments, the experimental data closely fit to the TE model indicated by the solid lines. It is clear that the dependence of the current variation on the metal species is more marked for the sample with treatment $\mathrm{C}$, which indicates that the $\phi_{\mathrm{B}}-\phi_{\mathrm{M}}$ relation was changed by the surface chemical treatment.

For all fabricated samples, $\phi_{\mathrm{B}}$ evaluated using the TE model is plotted as a function of $\phi_{\mathrm{M}}$ in Fig. 10(a). $\phi_{\mathrm{M}}$ is $4.28 \mathrm{eV}$ for $\mathrm{Ag}, 4.65 \mathrm{eV}$ for $\mathrm{Cu}, 5.10 \mathrm{eV}$ for $\mathrm{Au}, 5.15 \mathrm{eV}$ for Ni, and $5.65 \mathrm{eV}$ for $\mathrm{Pt}^{10)}$. The slope factor $S$ is the smallest for treatment $\mathrm{A}$ and the largest for treatment C. Although the $\phi_{\mathrm{M}}$ dependence of $\phi_{\mathrm{B}}$ changes with the surface treatment, the four fitted lines almost intersect at one point, $\phi_{\mathrm{M}}=5 \mathrm{eV}$. This point is considered to be the charge neutral point, $E_{\mathrm{CNL}}$, of $\mathrm{GaN}$, indicating that $E_{\mathrm{C}}-E_{\mathrm{CNL}}=\sim 0.9 \mathrm{eV}$, which is in good agreement with theoretical predictions ${ }^{36,37)}$ within the experimental error. The resultant electron affinity is $4.1 \mathrm{eV}$, which is in good agreement with the previously reported experimental data $^{38)}$. On the other hand, the evaluated $n$ values are plotted as a function of the metal work function in Fig. 10(b). The small $n$ values indicate that nearly ideal $I-V$ characteristics are obtained owing to the excellent quality of $\mathrm{GaN}$ on $\mathrm{GaN}$, which also indicates that the fit to the TE model is appropriate for the forward bias voltages.

For the reverse bias voltages, the TFE model was applied. In the TFE model ${ }^{3,35)}$, the $I-V$ characteristic for a nondegenerated semiconductor is given by ${ }^{15}$ )

$$
\begin{aligned}
& J=J_{S} \exp \left[\frac{q V}{k T}-\frac{q V}{E_{00} \operatorname{coth}\left(E_{00} / k T\right)}\right] \\
& J_{S}=\frac{A^{*} T \sqrt{\pi E_{00}}}{k} \sqrt{q\left(V-\phi_{f}\right)+\frac{q \phi_{B i}}{\cosh ^{2}\left(E_{00} / k T\right)}} \times \exp \left[-\frac{q \phi_{B i}}{E_{00} \operatorname{coth}\left(E_{00} / k T\right)}\right], \\
& E_{00}=\frac{q \hbar}{2} \sqrt{\frac{N_{D}}{m^{*} \varepsilon_{S}}}
\end{aligned}
$$


where $\phi_{\mathrm{f}}=E_{\mathrm{C}}-E_{\mathrm{FB}}$ and $\hbar$ is the reduced Planck constant. Examples of fitting results are shown in Fig. 11 for the samples with (a) treatment A and (b) treatment C. An excellent fit was achieved. The $\phi_{\mathrm{B}}$ values derived by fitting to the TFE model are plotted versus $\phi_{\mathrm{M}}$ in Fig. 12. $\phi_{\mathrm{B}}-\phi_{\mathrm{M}}$ relations similar to those in Fig. 10(a) can be seen. Although the $\phi_{\mathrm{B}}-\phi_{\mathrm{M}}$ plot for the sample with treatment E slightly deviates, the other plots nearly cross at around $\phi_{\mathrm{M}}$ $=5 \mathrm{eV}$, which again indicates that $E_{\mathrm{C}}-E_{\mathrm{CNL}}=\sim 0.9 \mathrm{eV}$.

\subsection{Correlation between GaN surface and metal/GaN interface}

The results described above indicate that the reduction in the amount of the surface oxide leads to a reduction of Fermi level pinning at the GaN surface and the subsequently formed metal/GaN interfaces. Figure 13(a) shows plots of $\phi_{\mathrm{B}}$ versus the XPS spectral area ratio $I_{\mathrm{O} 1 \mathrm{~s}(\mathrm{O}-\mathrm{Ga})} / I_{\mathrm{Ga}} \mathrm{d}$ for all SBDs. It can be clearly seen that the reduction in the amount of surface oxide leads to an increase in the variation range of $\phi_{\mathrm{B}}$ depending on the electrode metal. The slope factor $S$ is plotted against $I_{\mathrm{O} 1 \mathrm{~s}(\mathrm{O}-\mathrm{Ga})} / I_{\mathrm{Ga}}$ in Fig. 13(b). As the amount of surface oxide is reduced, $S$ increased nearly exponentially. These results imply that uncontrolled surface oxide should be removed to remove Fermi level pinning at the metal/GaN interface. It is highly likely that the surface oxide is a factor in interface disorder. According to the DIGS model, the origin of Fermi level pinning at the semiconductor surface, the metal/semiconductor interface, and furthermore, the insulator/semiconductor interface is the disorder of the chemical bonds at the surface and interfaces. The present experimental results can be explained by the DIGS model. In the present work, the disorder was quantified as the relative amount of surface oxide, i.e., $I_{\mathrm{O} 1 \mathrm{~s}(\mathrm{O}-\mathrm{Ga})} / I_{\mathrm{Ga}} \mathrm{d}$, to show that the reduction of disorder leads to the reduction of Fermi level pinning at the metal/GaN interfaces.

It should be noted that the position of Fermi level pinning on the untreated $\mathrm{GaN}$ surface derived by XPS and $E_{\mathrm{CNL}}$ determined from the $\phi_{\mathrm{B}}-\phi_{\mathrm{M}}$ plots for various surface treatments are roughly the same, i.e., $E_{\mathrm{C}}-0.9 \mathrm{eV}$ for both. $E_{\mathrm{CNL}}$ can be interpreted as the position of Fermi level pinning for the strongly pinned metal/semiconductor interface. Therefore, it can be concluded that strong pinning commonly occurs at $E_{\mathrm{C}}-0.9 \mathrm{eV}$ for both the GaN surface and the metal/GaN interface. Furthermore, on the basis of Figs. 10(a) and 12 , the strong pinning point or $E_{\mathrm{CNL}}$ locates at $\sim 5 \mathrm{eV}$ from the vacuum level. Again, this result is in agreement with the prediction by the DIGS model ${ }^{8)}$, which insists that $E_{\mathrm{CNL}}$ should locate at around $5 \mathrm{eV}$ under the vacuum level for all semiconductors. 


\section{Conclusions}

The effect of chemical surface treatment on the uncontrolled surface oxide at a GaN surface and on Fermi level pinning at metal/GaN interfaces was investigated for a $\mathrm{GaN}$ epitaxial layer grown on a GaN substrate. XPS study revealed that surface band bending was reduced by the reduction in the amount of uncontrolled surface oxide. It is highly likely that the decay of $Q_{\mathrm{S}}$ resulting in an $E_{\mathrm{FS}}$ shift toward $E_{\mathrm{C}}$ occurred as the amount of surface oxide was reduced. The electrical measurement of completed SBDs revealed that the $\phi_{\mathrm{B}}-\phi_{\mathrm{M}}$ relation was greatly affected by the surface treatments. It was shown that the reduction in the amount of surface oxide led to a reduction in Fermi level pinning, resulting in an increase in the slope factor of the $\phi_{\mathrm{B}}-\phi_{\mathrm{M}}$ relation. The observed phenomena can be explained by the DIGS model.

\section{Acknowledgments}

This work was partially supported by JSPS KAKENHI Grant Number JP16H06421. 


\section{References}

1) T. Kachi, Jpn. J. Appl. Phys. 53, 100210 (2014).

2) H. Amano et al., J. Phys. D: Appl. Phys. 51, 163001 (2018).

3) S. M. Sze and K. K. Ng, Physics of Semiconductor Devices (Wiley, Hoboken, NJ, 2007) 3rd ed., Chap. 3.

4) W. J. Bardeen, Phys. Rev. 71, 717 (1947).

5) A. M. Cowley and S.M. Sze, J. Appl. Phys. 36, 3212 (1965).

6) V. Heine, Phys. Rev. A 138, 1689 (1965).

7) W. E. Spicer, I. Lindau, P.R. Skeath and C.Y. Su : J. Vac. Sci. Technol. 17, 1019 (1980).

8) H. Hasegawa and H. Ohno, J. Vac. Sci. Technol. B 4, 1130 (1986).

9) E. V. Kalinina, N. I. Kuznetsov, V. A. Dmitriev, K. G. Irvine, and C. H. Carte, JR., J. Electron. Mater. 25, 831 (1996).

10) A. C. Schmitz, A. T. Ping, M. Asif Khan, Q. Chen. J. W. Yang, and I. Adesida, J. Electron. Mater. 27, 255 (1998).

11) V. M. Bermudez, J. Appl. Phys. 86, 1170 (1999).

12) H. Hasegawa, Y. Koyama, and T. Hashizume, Jpn. J. Appl. Phys. 38, 2634 (1999).

13) J. K. Kim and J.-L. Lee, J. Electrochemical Soc. 150, G209 (2003).

14) K. M. Tracy, P. J. Hartlieb, S. Einfeldt, R. F. Davis, E. H. Hurt, and R. J. Nemanich, J. Appl. Phys. 94, 3939 (2003).

15) S. Oyama, T Hashizume, and H. Hasegawa, Appl. Surf. Sci. 190, 322 (2002).

16) H. Hasegawa and S. Oyama, J. Vac. Sci. Technol. B 20, 1647 (2002).

17) J. Kotani, S. Kasai, T. Hashizume, and H. Hasegawa, J. Vac. Sci. Technol. B 23, 1799 (2005).

18) J. Suda, K. Yamaji, Y. Hayashi, T. Kimoto, K. Shinoyama, H. Namita, and S. Nagao, Appl. Phys. Express 3, 101003 (2010).

19) T. Maeda, M. Okada, M. Ueno, Y. Yamamoto, T. Kimoto, M. Horita, and J. Suda, Appl. Phys. Express 10, 051002 (2017).

20) M. Akazawa and T. Hasezaki, Phys. Status Solidi B 255, 1700381 (2018).

21) H. Imadate, T. Mishima, and K. Shiojima, Jpn. J. Appl. Phys. 57, 04 FG13 (2018)

22) K. Isobe, and M. Akazawa, AIP Advances 8, 115011 (2018)

23) Y.-J. Lin and C.-T. Lee, Appl. Phys. Lett. 77, 3986 (2000)

24) I. Waki, H. Fujioka, K. Ono, M. Oshima, H. Miki, and A. Fukizawa, Jpn. J. Appl. Phys. 39, $4451(2000)$

25) H. Ishikawa, S. Kobayashi, Y. Koide, S. Yamasaki, S. Nagai, J. Umezaki, M. Koide, M. 
Murakami, J. Appl. Phys. 81, 1315 (2001).

26) I. Shalish, Y. Shapira, L. Burstein, and J. Salzman, J. Appl. Phys. 89, 390 (2001).

27) J. K. Kim and J.-L. Lee, J. Electrochemical Soc. 150, G209 (2003).

28) Y. Irokawa, T. T. Suzuki, K. Yuge, A. Phi, T. Nabatame, K. Kimoto, T. Ohnishi, K. Mitsuishi, and Y. Koide, Jpn. J. Appl. Phys. 56, 128004 (2017).

29) J. H. Dycus, K. J. Mirrielees, E. D. Grimley, R. Kirste, S. Mita, Z. Sitar, R. Collazo, D. L. Irving, and J. M. Lebeau, Appl. Mater. Interfaces 10, 10607 (2018).

30) Y. Irokawa, K. Mitsuishi, T. T. Suzuki, K. Yuge, A. Ohi, T. Nabatame, T. Ohnishi, K. Kimoto, and Y. Koide, Jpn. J. Appl. Phys. 57, 098003 (2018).

31) K. Mitsuishi, K. Kimoto, Y. Irokawa, T. Suzuki, K. Yuge, T. Nabatame, S. Takashima, K. Ueno, M. Edo, K. Nakagawa, and Y. Koide, Jpn. J. Appl. Phys. 56, 110312 (2017).

32) T. Yamamoto, N. Taoka, A. Ohta, N. X. Truyen, H. Yamada, T. Takahashi, M. Ikeda, K. Makihara, and S. Miyazaki, Jpn. J. Appl. Phys. 57, 06JE01 (2018).

33) T. Yamada, D. Terashima, M. Nozaki, H. Yamada, T. Takahashi, M. Shimizu, A. Yoshigoe, T. Hosoi, T. Shimura, and H. Watanabe, Jpn. J. Appl. Phys. 58, SCCD06 (2019).

34) M. Kodama, M. Sugimoto, E. Hayashi, N. Soejima, O. Ishiguro, M. Kanechika, K. Itoh, H. Ueda, T. Uesugi, and T. Kachi, Appl. Phys. Express 1, 021104 (2008).

35) P. A. Padovani and R. Stratton, Solid-State Electron. 9, 695 (1966).

36) C. G. Van de Walle and J. Neugebauer, Nature 423, 626 (2003).

37) W. Mönch, J. Appl. Phys. 109, 113724 (2011).

38) J. I. Pankove and H. Schade, Appl. Phys. Lett. 25, 53 (1974). 


\section{Figure Captions}

Fig. 1. Schematic diagram of $\phi_{\mathrm{B}}-\phi_{\mathrm{M}}$ plot indicating degree of Fermi level pinning at metal/semiconductor interface.

Fig. 2. Structure of completed SBD.

Fig. 3. XPS O 1s spectra from several $\mathrm{GaN}$ surfaces indicating the effect of chemical treatments. The intensity is normalized to that of Ga $3 \mathrm{~d}$ peak area for each treatment. [Data for untreated, treatment $\mathrm{A}$, and treatment $\mathrm{C}$ samples are taken from our previous report ${ }^{22)}$; licensed under a Creative Commons Attribution (CC BY) license.]

Fig. 4. Shapes of $\mathrm{Ga} 3 \mathrm{~d}$ spectra of the GaN surfaces without any treatments and with treatment $\mathrm{C}$, where the two peaks were aligned by height. The peak of the latter was shifted to overlap with that of the former.

Fig. 5. XPS VBM spectra from several GaN surfaces indicating the effect of chemical treatments.

Fig. 6. E Es position plotted versus $I_{\mathrm{O} 1 \mathrm{~s}(\mathrm{O}-\mathrm{Ga})} / I_{\mathrm{Ga} 3 \mathrm{~d}}$. The solid line is the fitted line based on Eqs. (3), (4) and fitting result in Fig. 7. [Data for untreated, treatment A and treatment C samples are taken from our previous report ${ }^{22)}$; licensed under a CC BY license.]

Fig. 7. $Q \mathrm{~s}$ plotted versus $I_{\mathrm{O} 1 \mathrm{~s}(\mathrm{O}-\mathrm{Ga})} / I_{\mathrm{Ga}} \mathrm{d}$. The solid line is the fitted line (exponential approximation).

Fig. 8. $I-V$ characteristics of SBDs fabricated with (a) treatment $\mathrm{A}$, (b) treatment $\mathrm{B}$, (c) treatment $\mathrm{C}$, and (d) treatment E. [Data for Ag and Ni electrodes of treatment $\mathrm{C}$ sample are taken from our previous report ${ }^{22)}$; licensed under a CC BY license.]

Fig. 9. $I-V$ characteristics under forward bias voltage for SBDs with (a) treatment A and (b) treatment C. Solid lines are fitted lines based on the TE model.

Fig. 10. (a) $\phi_{\mathrm{B}}-\phi_{\mathrm{M}}$ plots derived from forward bias region for SBDs fabricated with several chemical treatments. Solid lines are fitted lines. (b) Plot of $n$ versus $\phi_{\mathrm{M}}$ for SBDs fabricated with several chemical treatments. [Data for treatment $\mathrm{A}$ and treatment $\mathrm{C}$ samples are partially taken from our previous report ${ }^{22)}$; licensed under a CC BY license]

Fig. 11. $I-V$ characteristics under reverse bias voltage for SBDs with (a) treatment $\mathrm{A}$ and 
(b) treatment C. Solid lines are fitted lines based on the TFE model.

Fig. 12. $\phi_{\mathrm{B}}-\phi_{\mathrm{M}}$ plots derived from reverse bias region for SBDs fabricated with several chemical treatments. Solid lines are fitted lines.

Fig. 13. Plots of (a) $\phi_{\mathrm{B}}$ of all SBDs versus $I_{\mathrm{O} 1 \mathrm{~s}(\mathrm{O}-\mathrm{Ga})} / I_{\mathrm{Ga} d \mathrm{~d}}$ measured by XPS and (b) $S$ for several chemical treatments versus $I_{\mathrm{O} 1 \mathrm{~s}(\mathrm{O}-\mathrm{Ga})} / I_{\mathrm{Ga}} \mathrm{d}$. Solid marks are data derived from the forward bias side, while open marks are those from the reverse bias side. 
Table I. Details of surface chemical treatments.

\begin{tabular}{cc}
\hline Designation & Details \\
\hline A & photolithography with dipping in TMAH-based developer for $25 \mathrm{~s}$ \\
B & BHF treatment after treatment A \\
C & HCl treatment after treatment A \\
D & photolithography with dipping in TMAH-based developer for $1 \mathrm{~min}$ \\
E & $\mathrm{HCl}$ treatment after treatment D \\
\hline
\end{tabular}




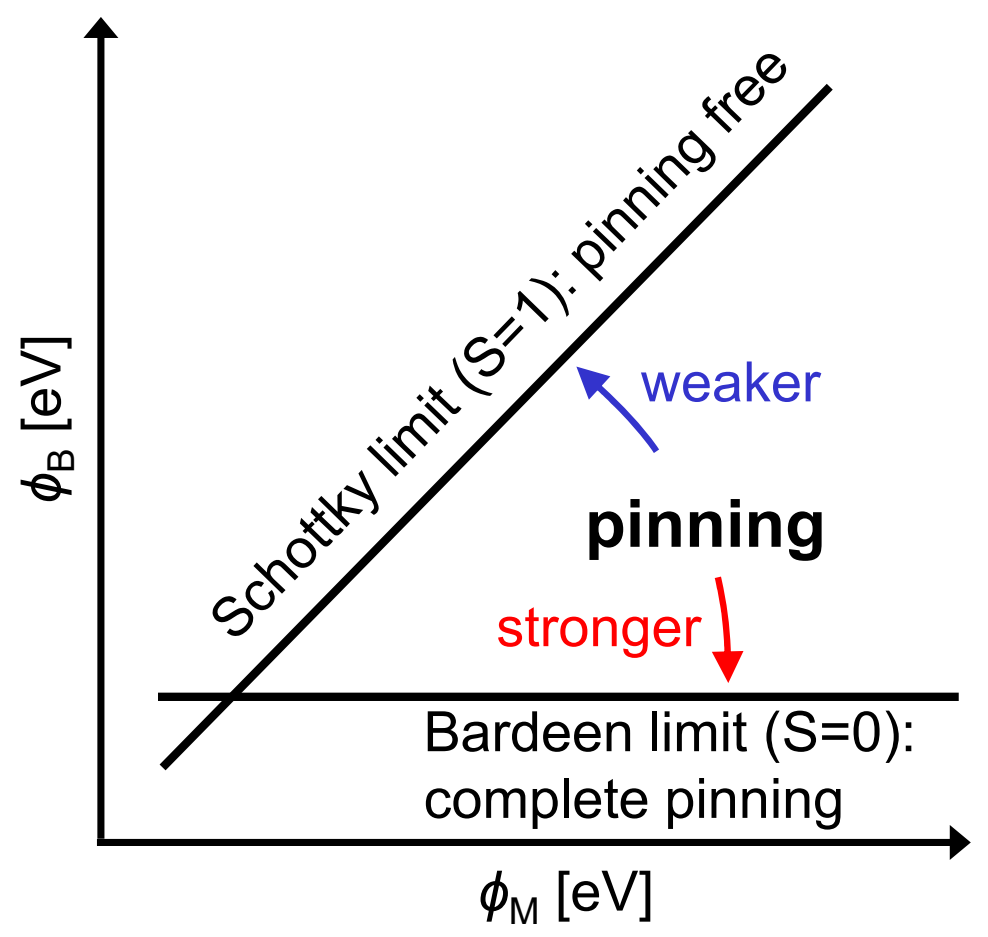

Fig.1.

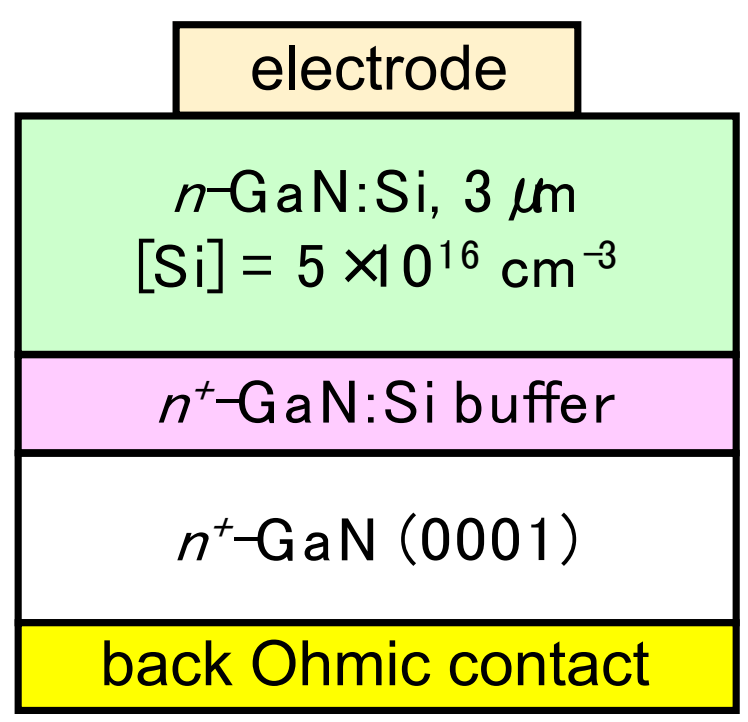

Fig. 2. 

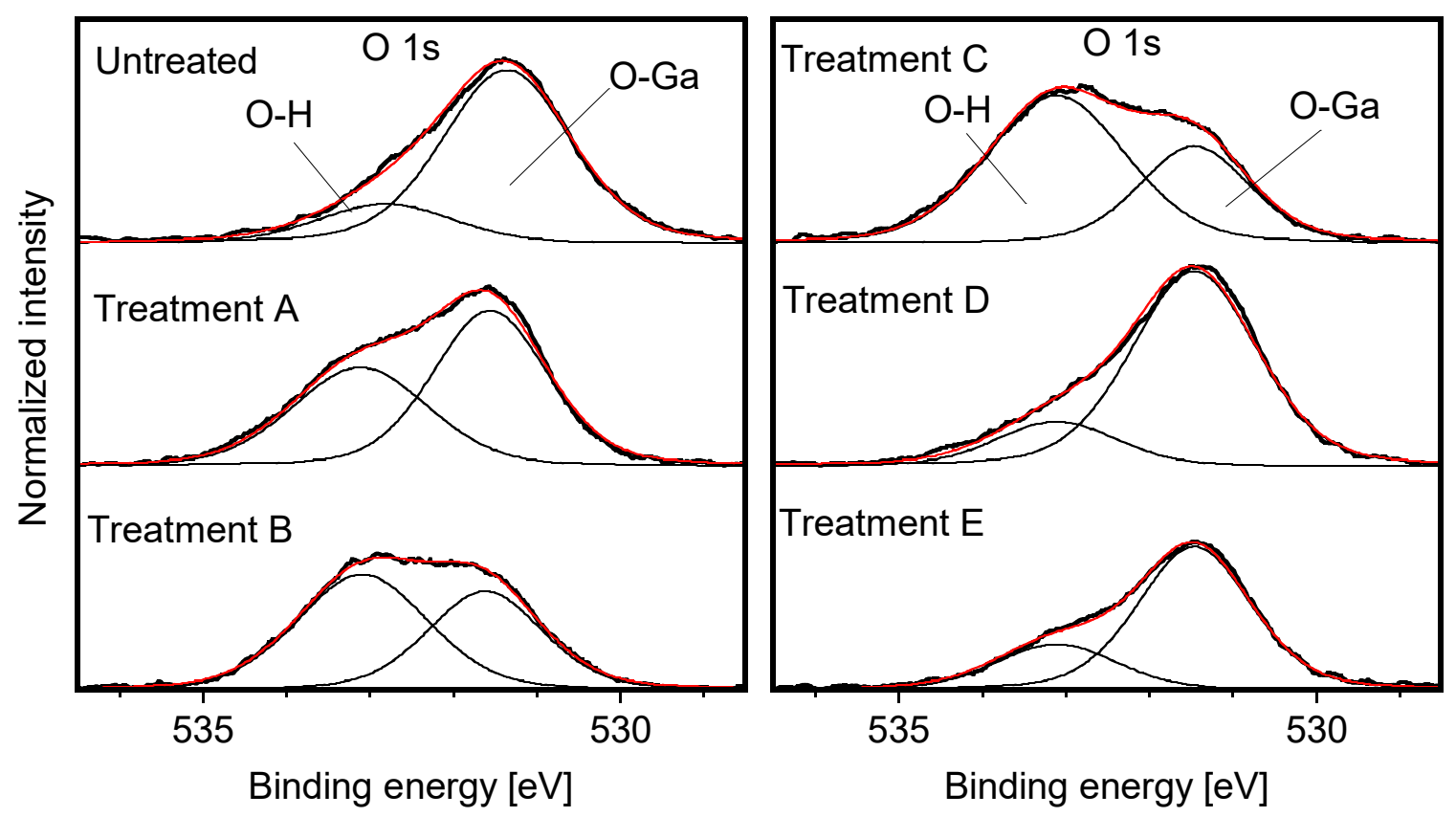

Fig. 3.

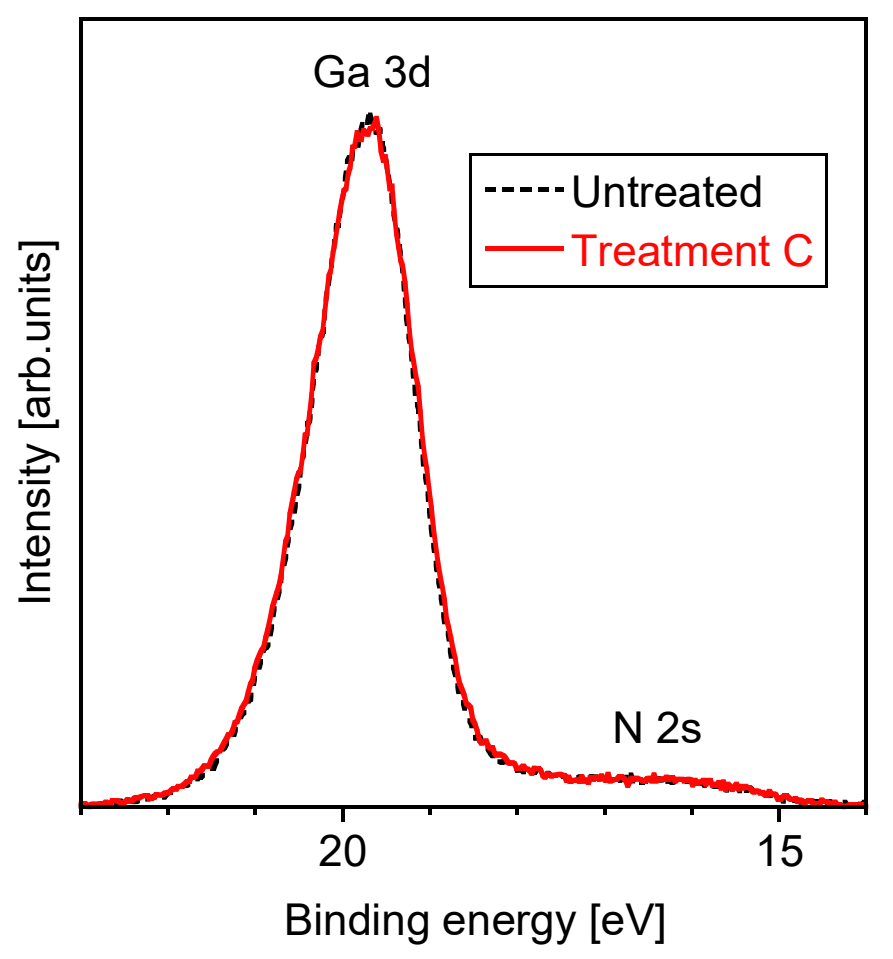

Fig. 4. 


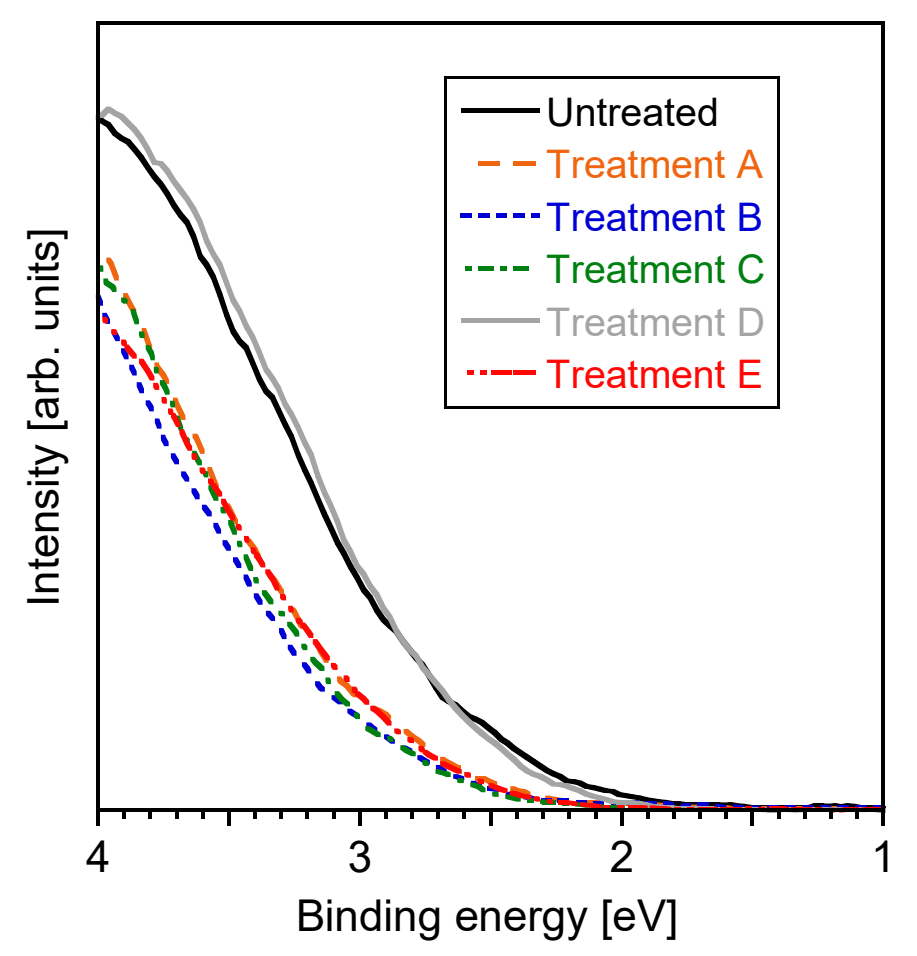

Fig. 5. 


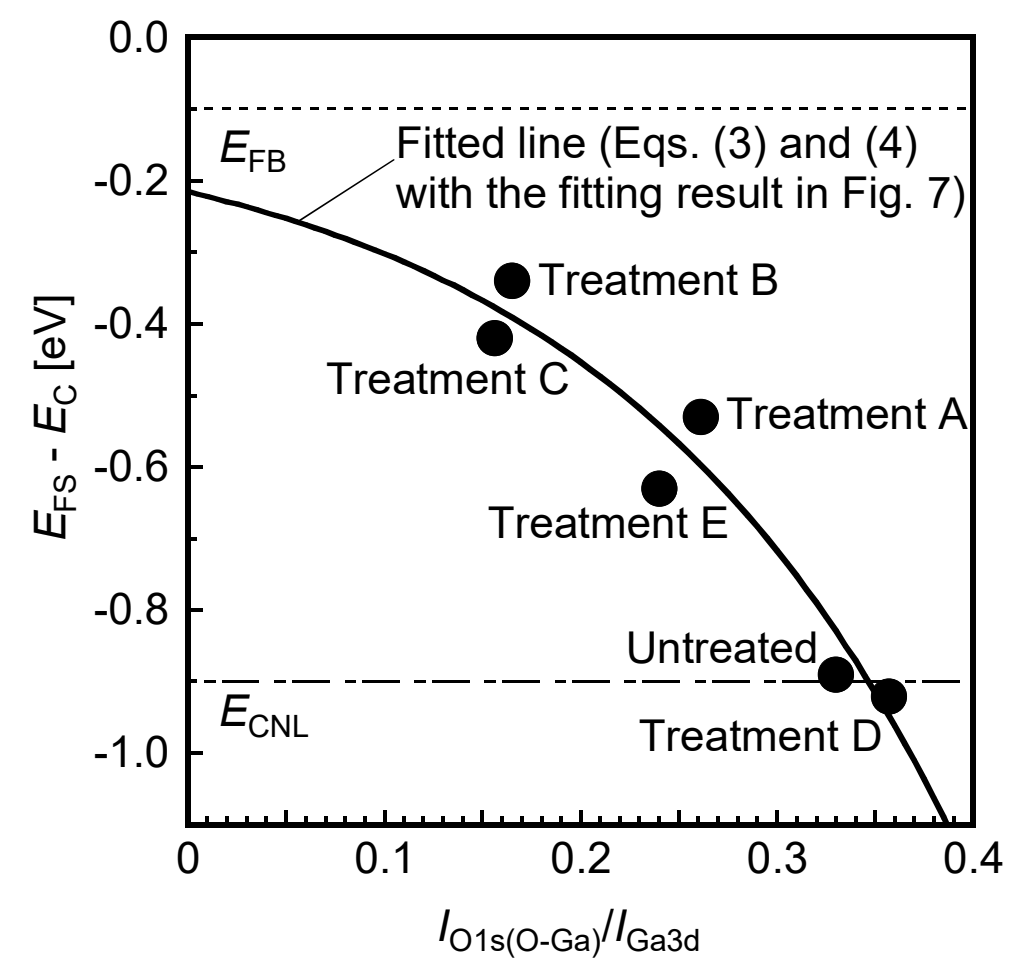

Fig. 6.

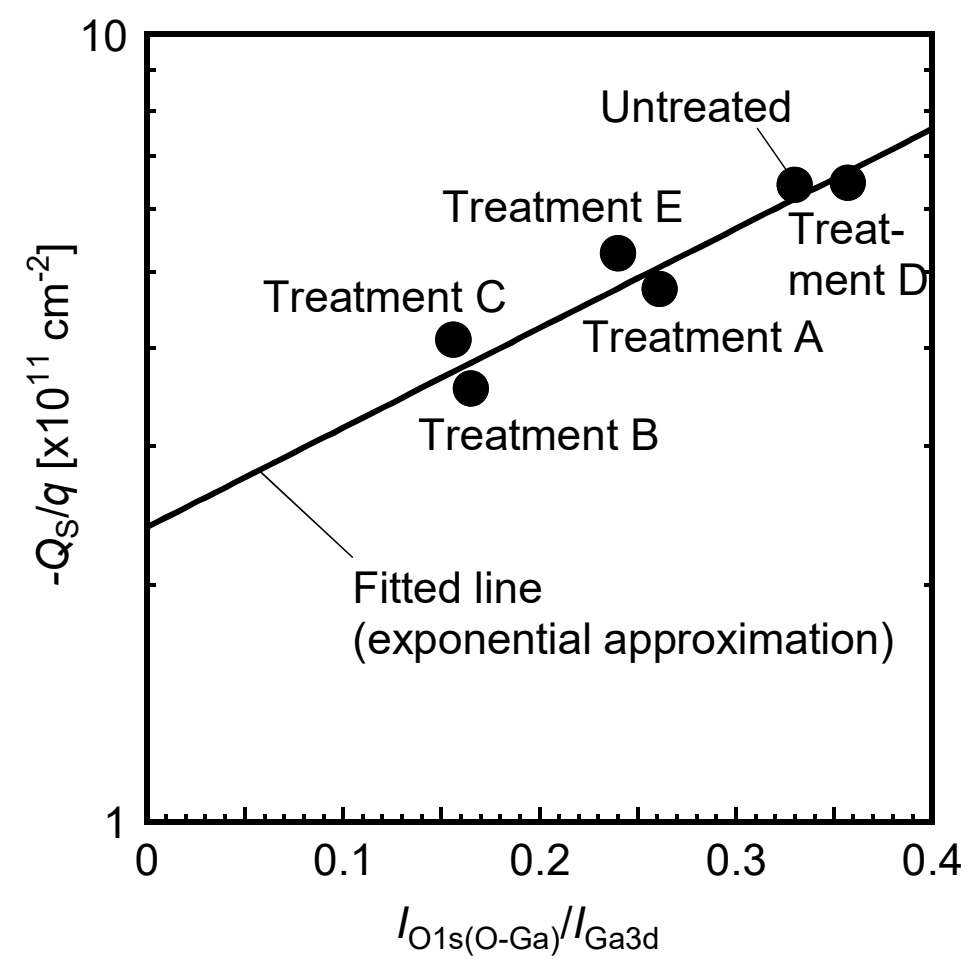

Fig. 7. 

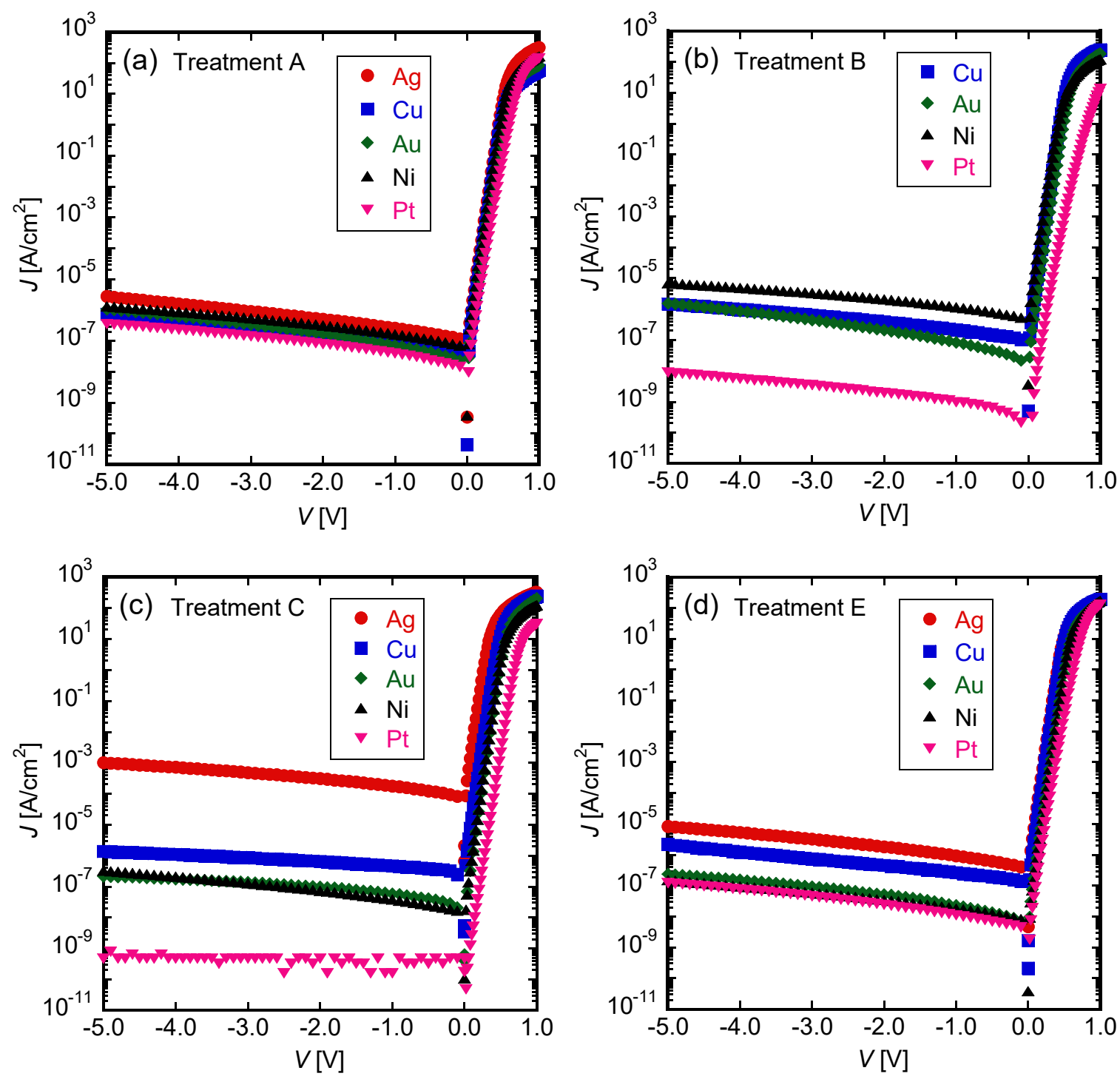

Fig. 8. 

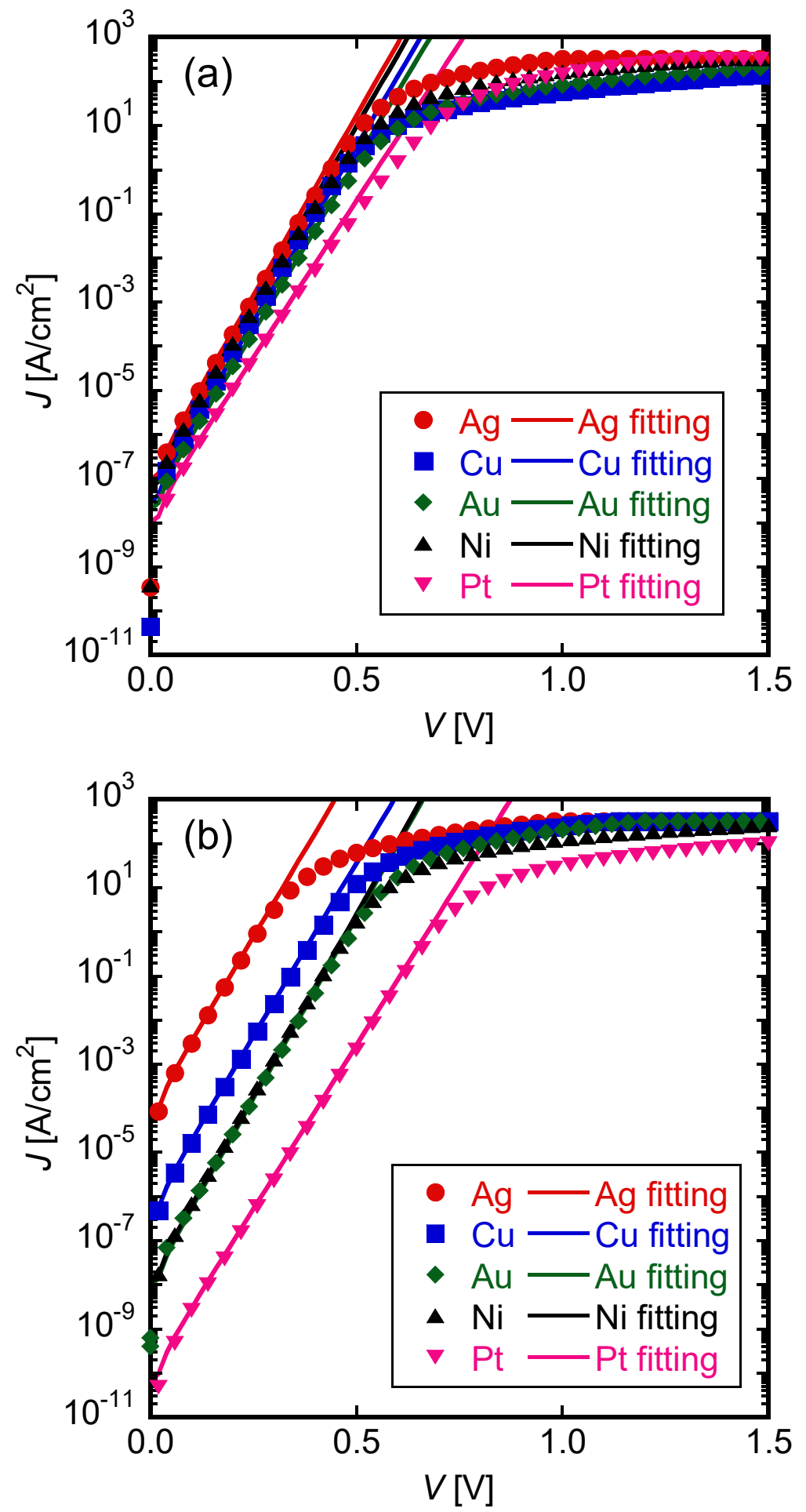

Fig. 9. 

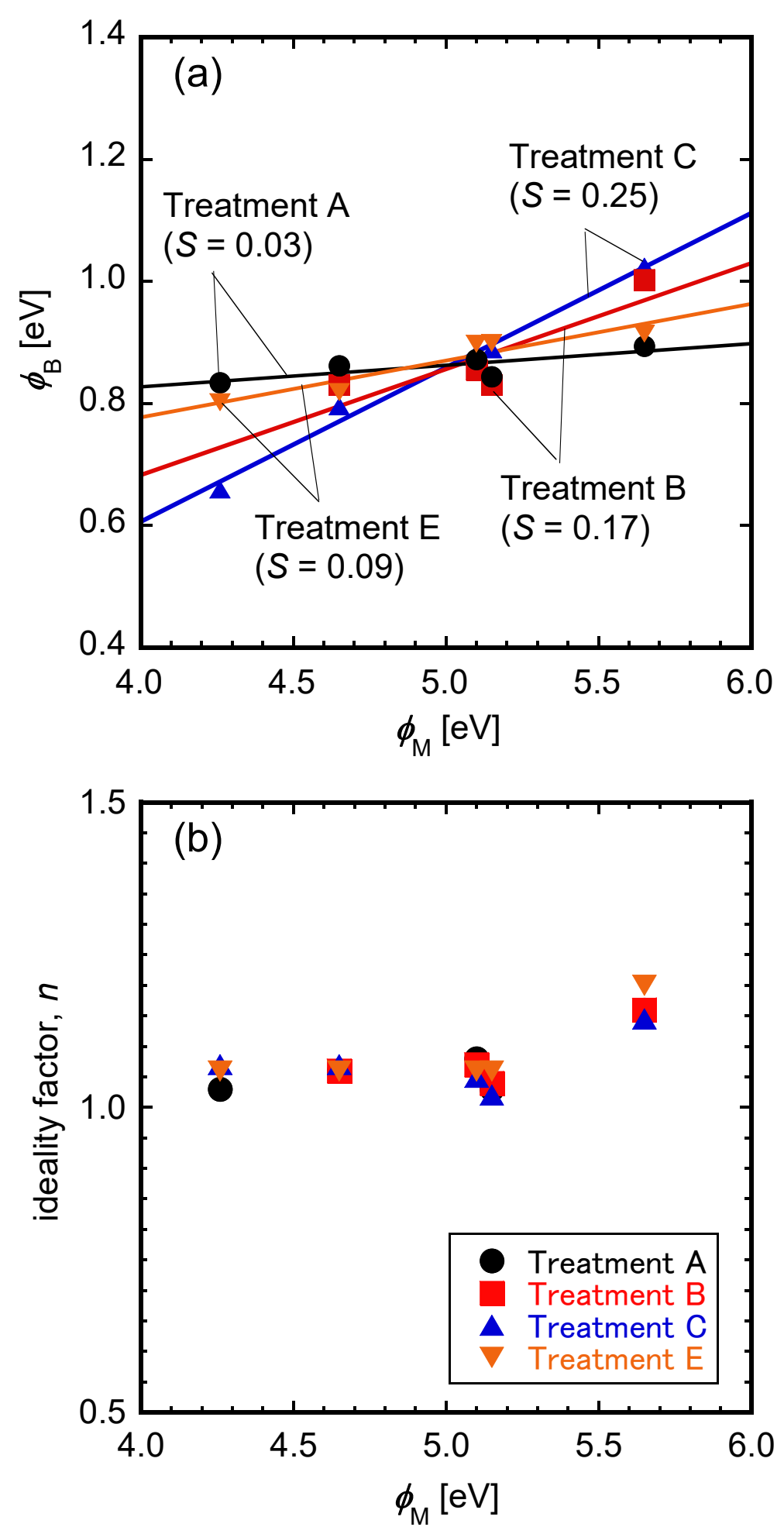

Fig. 10. 

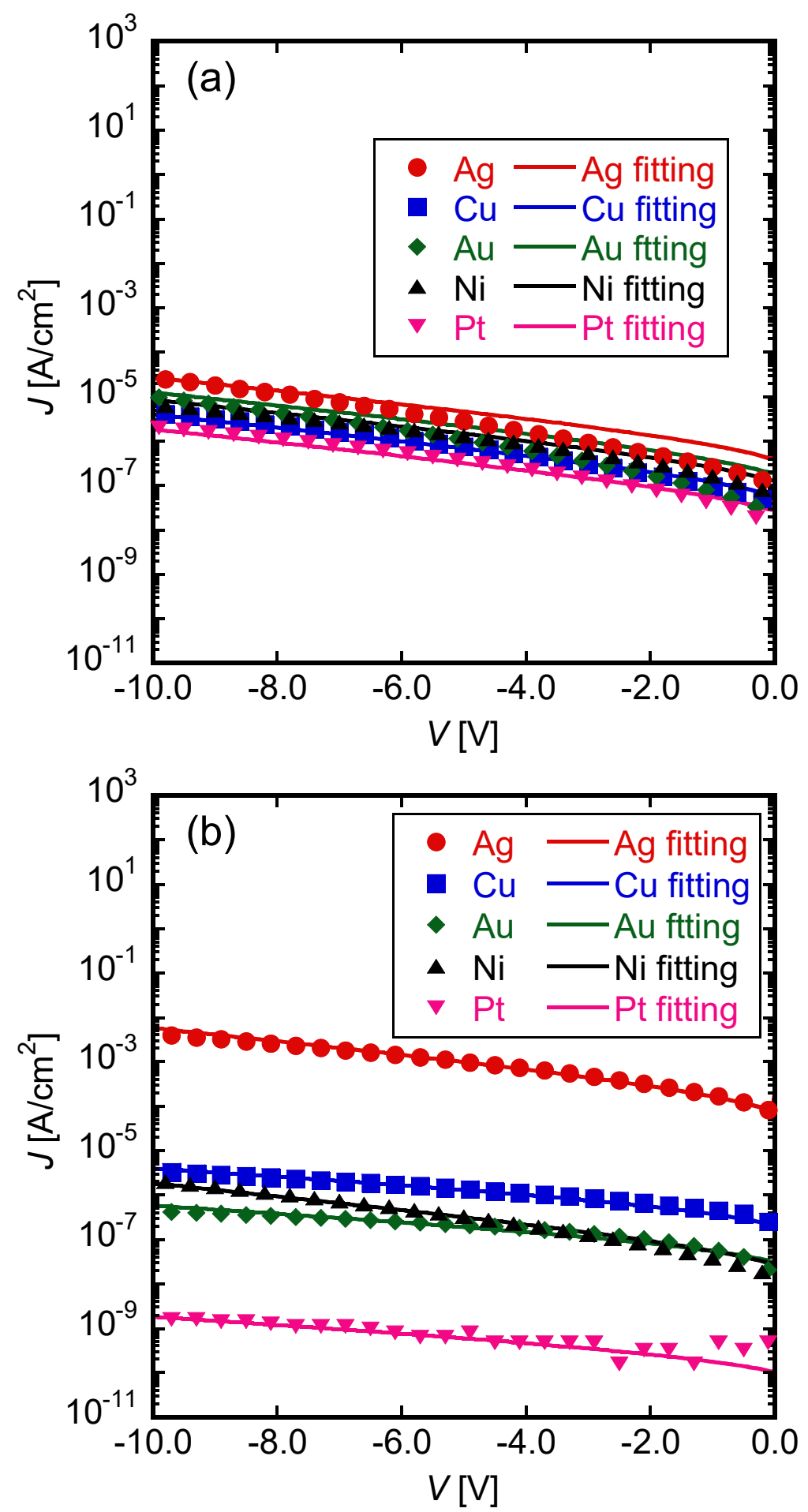

Fig. 11. 


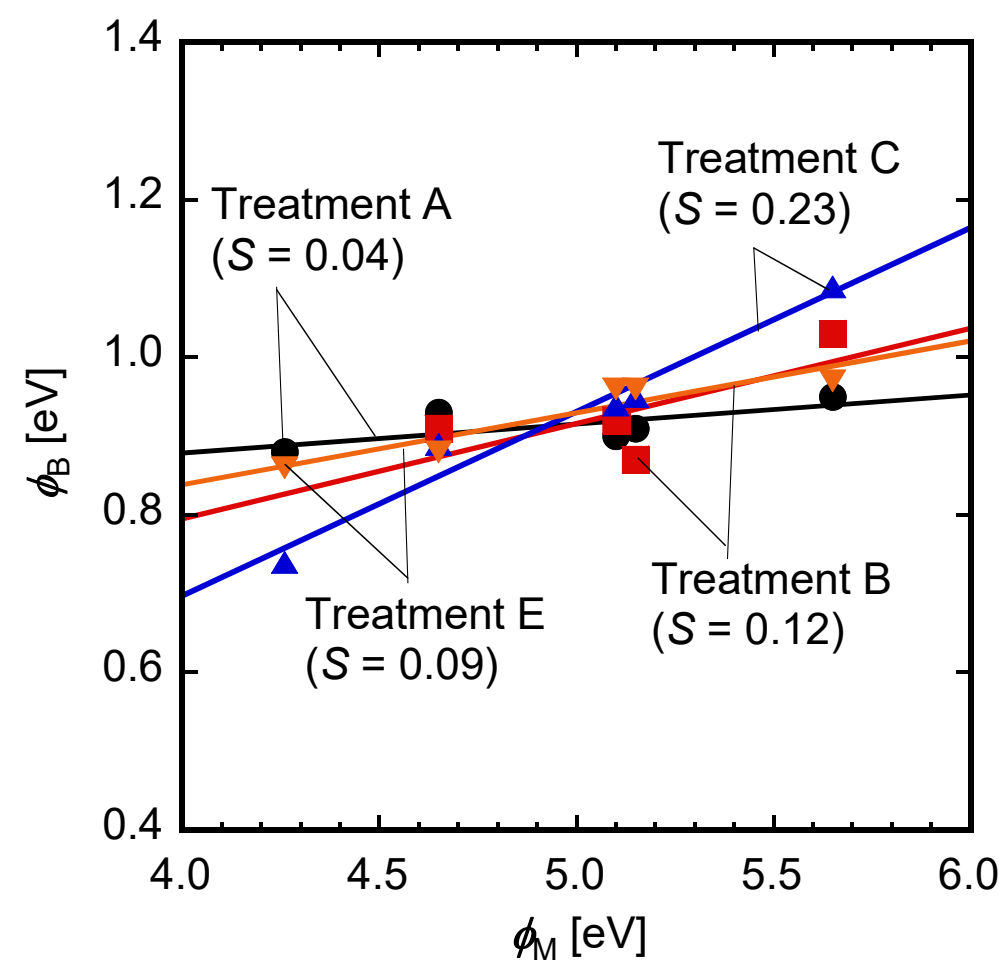

Fig. 12. 

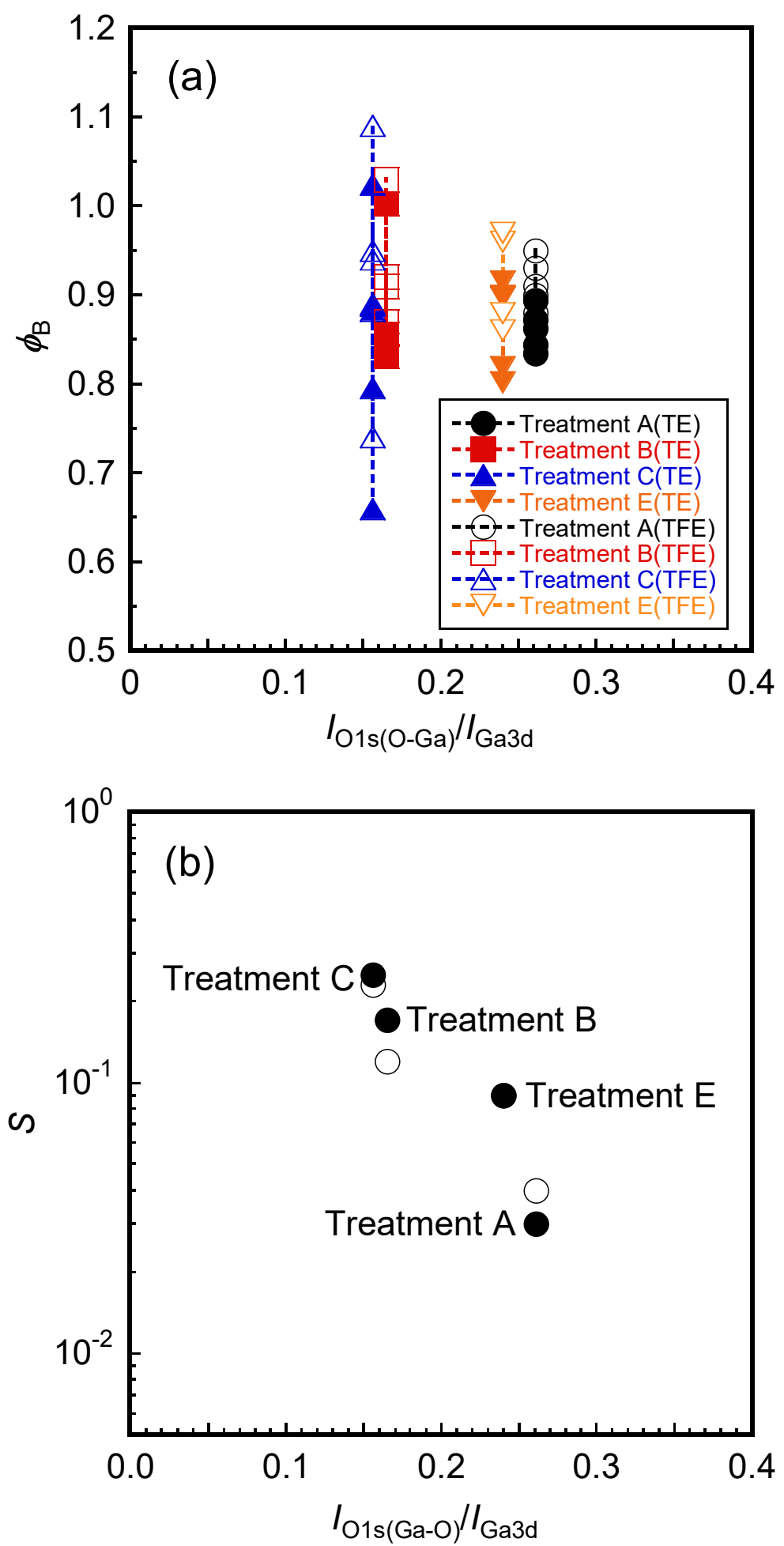

Fig. 13. 